
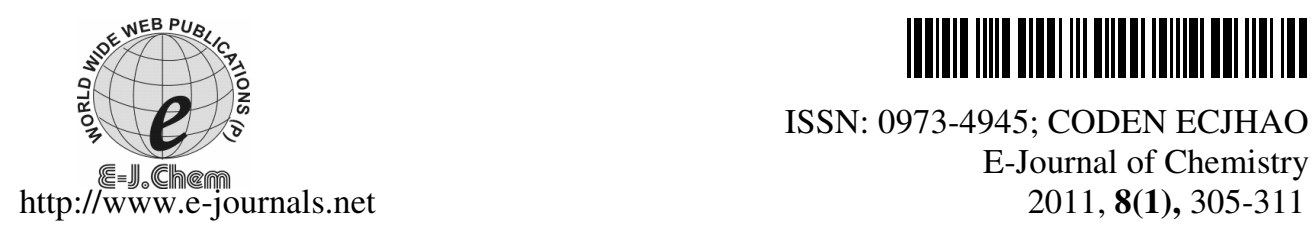

ISSN: 0973-4945; CODEN ECJHAO

E-Journal of Chemistry

2011, 8(1), 305-311

\title{
Microwave Assisted Synthesis and Antimicrobial Evaluation of Schiff Bases of Indole-3-aldehyde
}

\author{
PRIYANKA KAMARIA*, N. KAWATHEKAR and PRERNA CHATURVEDI
}

Department of Pharmacy

Shri Govindram Seksaria Institute of Technology and Science

23-Park Road, Indore- 452003, (M.P.) India

kamaria.priyanka@gmail.com

Received 23 April 2010; Accepted 17 June 2010

\begin{abstract}
In order to develop new antimicrobial agents, a series of Schiff bases of indole-3-aldehyde were synthesized by microwave assisted synthesis by taking DMF as solvent and evaluated for their antimicrobial activity. All the synthesized compounds were characterized by IR, ${ }^{1} \mathrm{H}$ NMR and mass spectral analysis. All compounds were tested against five gram positive and five gram negative bacterial strains and one fungal strain. All compounds exhibited better activity against gram positive strains than against gram negative strains and the compounds were found more active against S.aureus and B.subtilis.
\end{abstract}

Keywords: Antibacterial, Antifungal, Schiff base, Indole-3-aldehyde.

\section{Introduction}

A Schiff base (or azomethine) is a functional group that contains a carbon nitrogen double bond with the nitrogen atom connected to an aryl or alkyl group but not hydrogen ${ }^{1}$. Schiff bases are usually synthesized from the condensation of primary amines and active carbonyl group $^{2}$. Schiff bases have been reported to possess antimicrobial properties ${ }^{3-7}$. Schiff bases are characterized by the $-\mathrm{N}=\mathrm{CH}-$ (imines) group which is important for elucidating the mechanism of transamination and racemisation reactions in biological systems and are also known to have biological activities such as antimicrobial ${ }^{8}$ antifungal $^{9}$, antitumor ${ }^{10}$ and herbicidal ${ }^{11}$ activity. Indole derivatives found to possess antibacterial ${ }^{12}$, anticonvulsant ${ }^{13}$ and antihypertensive activity. These observations led to the conception that Schiff bases of indole-3-aldehyde would possess potential antimicrobial properties. Microwave assisted organic synthesis (MAOS) accelerate the course of many organic reactions, producing high yields and higher selectivity, lower quantities of side products and consequently, easier work-up and purification of the products ${ }^{14}$. In the present study a series of Schiff base of Indole-3-aldehyde were synthesized by use of conventional microwave and characterized by 
IR, ${ }^{1} \mathrm{H}$ NMR and mass spectroscopy. The compounds were screened for antibacterial and antifungal activities. The minimum inhibitory concentrations of the compounds were also determined by serial dilution method.

Indole-3-aldehyde on condensation with different aromatic or aliphatic amine in presence of DMF as solvent yields Schiff bases (Scheme 1).

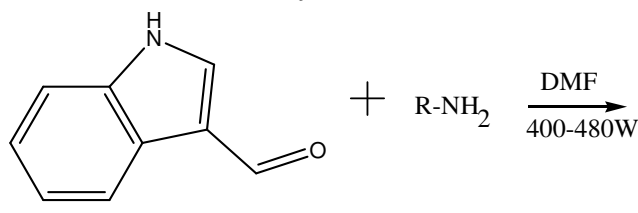

Indole-3-aldehyde

Alkyl/aryl amine

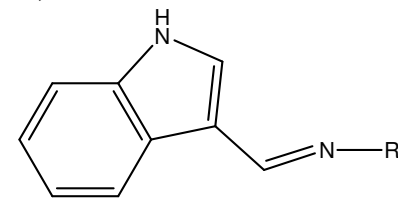

Schiff base of Indole-3-aldehyde

\section{Experimental}

Figure 1. Scheme for the synthesis of Schiff bases

A mixture of 2 mmole of indole-3-aldehyde and 2 mmole of different aryl or alkyl amines was taken and triturated in a mortar pestle. Then above mixture was transferred to a vessel which was then kept in microwave for synthesis. 4 to $5 \mathrm{~mL}$ of DMF was also added to mixture before putting it in microwave. Microwave was run at $400-480 \mathrm{~W}$ for different time for different reaction mixtures. Reaction completion was monitored continuously after each run by TLC. Then product was washed with ethanol, solvent was evaporated, dried and recrystalized with ethanol. Structures, time in microwave assisted synthesis and \% yield are given in Table 1

Table1. Structures, time in microwave assisted synthesis and \% yield of synthesized compounds.

\begin{tabular}{|c|c|c|c|}
\hline $\begin{array}{l}\text { Compound } \\
\text { code }\end{array}$ & $\mathrm{R}$ & $\begin{array}{l}\text { Time in microwave assisted } \\
\text { synthesis, min. }\end{array}$ & $\%$ yield \\
\hline PK1 & & 4 & 71.52 \\
\hline PK2 & & 6 & 64.2 \\
\hline PK3 & $\mathrm{C}_{4} \mathrm{H}$ & 3 & 71.0 \\
\hline PK4 & & 5 & 65.6 \\
\hline PK5 & & 7 & 68.2 \\
\hline PK6 & & 12 & 64.5 \\
\hline PK7 & & 9 & 62.3 \\
\hline PK8 & & 5 & 62.7 \\
\hline
\end{tabular}




\begin{tabular}{|c|c|c|c|}
\hline PK9 & & 7 & 72.6 \\
\hline PK10 & & 10 & 53.3 \\
\hline PK11 & & 5 & 74.8 \\
\hline PK12 & & 11 & 55.5 \\
\hline PK13 & & 12 & 61.6 \\
\hline PK14 & & 15 & 51.7 \\
\hline
\end{tabular}

\section{Physicochemical and spectroscopic determination of synthesized compounds}

The melting points were taken in open capillary tube and were uncorrected. Reactions were monitored by thin layer chromatography using silica gel- $\mathrm{GF}_{254}$ as adsorbent on glass plate. The spots were applied on silica gel plate and the plate was run in (ethyl acetate: hexane (3:7)) in a closed chamber. The spots on the plate were detected in UV cabinet. The differences in $R_{f}$ value between starting compound and product were indicative of the conversion of starting compound into the product.

IR spectra were recorded on FTIR-8400F model in $\mathrm{KBr}$. NMR spectra were recorded on Broker A VANCE DPX 300 instrument using TMS as internal reference and chemical shift value are expressed in delta units. The mass spectra (ESI) were recorded on Waters @Micromass $Q$-Tof Micro. It showed fragmentation pattern as $m / z$ values. All physical and spectral data are given in Table 2.

Table 2. Physical and spectroscopic data of synthesized compounds

\begin{tabular}{|c|c|}
\hline $\begin{array}{c}\text { Compd } \\
\text { code }\end{array}$ & Physical and spectral data \\
\hline PK1 & $\begin{array}{l}\text { Yield:72.6\%; Rf: } 0.63\{\text { ethyl acetate: hexane }(3: 7)\} ; \mathrm{M} . \mathrm{P}: 120-125{ }^{\circ} \mathrm{C} ; \mathrm{IR}\left(\mathrm{KBr} \mathrm{cm}^{-1}\right) \\
: 1570(\mathrm{C}=\mathrm{C}), 1682.11(\mathrm{C}=\mathrm{N}), 3042.32(\mathrm{C}-\mathrm{H}), 1244.86(\mathrm{C}-\mathrm{N}), 2852.41(\mathrm{~N}=\mathrm{CH}) ; \mathrm{NMR}:{ }^{1} \mathrm{H} \\
\mathrm{NMR}\left(400 \mathrm{MHz}, \mathrm{CDCl}_{3}\right) \delta(\mathrm{ppm}): 6.87-7.22(\mathrm{~m}, 10 \mathrm{H}, \mathrm{ArH}), 8.51(\mathrm{~s}, 1 \mathrm{H} ; \mathrm{N}=\mathrm{CH}), 7.60 \\
(\mathrm{~s}, 1 \mathrm{H}, \mathrm{CH}) ;(\mathrm{m}+1) 221.1\end{array}$ \\
\hline PK2 & 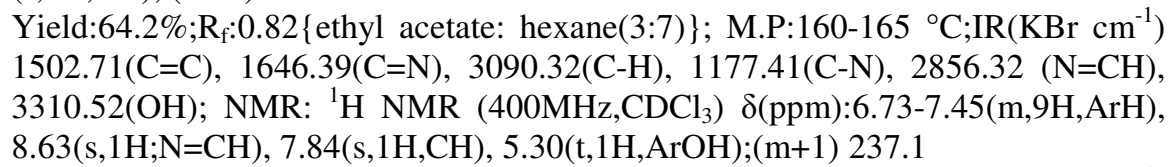 \\
\hline PK3 & $\begin{array}{l}\text { Yield:71.0\%; } \mathrm{R}_{\mathrm{f}}: 0.73\{\text { ethyl acetate: hexane }(3: 7)\} ; \mathrm{M} . \mathrm{P}: 170-175{ }^{\circ} \mathrm{C} ; \mathrm{IR}\left(\mathrm{KBr} \mathrm{cm}^{-1}\right) \\
1520.34(\mathrm{C}=\mathrm{C}), 1676.34(\mathrm{C}=\mathrm{N}), 3108.19(\mathrm{C}-\mathrm{H}), 1243.06(\mathrm{C}-\mathrm{N}), 2859.34(\mathrm{~N}=\mathrm{CH}), 1444.33 \\
\left(\mathrm{CH}_{3}\right) \text { bend; NMR: }{ }^{1} \mathrm{H} \quad \mathrm{NMR}\left(400 \mathrm{MHz}, \mathrm{CDCl}_{3}\right) \delta(\mathrm{ppm}): 7.28-7.41 \quad(\mathrm{~m}, 5 \mathrm{H}, \mathrm{ArH}), 8.61 \\
(\mathrm{~s}, 1 \mathrm{H} ; \mathrm{N}=\mathrm{CH}), 7.68(\mathrm{~s}, 1 \mathrm{H}, \mathrm{CH}), 2.4\left(\mathrm{~s}, 2 \mathrm{H}, \mathrm{CH}_{2}\right), 1.7\left(\mathrm{~m}, 5 \mathrm{H}, \mathrm{CH}_{2}\right), 1.4\left(\mathrm{~m}, 6 \mathrm{H}, \mathrm{CH}_{2}\right) ;(\mathrm{m}+1) 201.2\end{array}$ \\
\hline PK4 & $\begin{array}{l}\text { Yield:65..6\%; Rf: } 0.72 \text { \{ethyl acetate: hexane }(3: 7)\} ; \mathrm{M} . \mathrm{P}: 115-120{ }^{\circ} \mathrm{C} ; \mathrm{IR}\left(\mathrm{KBr} \mathrm{cm}^{-1}\right) \\
\text { 1618.17(C=C), 1682.41(C=N), 2943.37, 2903(C-H), 1244.92(C-N), 2854.56(N=CH), } \\
\text { 1072.34(C-O); NMR: }{ }^{1} \mathrm{H} \mathrm{NMR}\left(400 \mathrm{MHz}, \mathrm{CDCl}_{3}\right) \delta: 6.81-7.29(\mathrm{~m}, 9 \mathrm{H}, \mathrm{ArH}), 8.53 \\
(\mathrm{~s}, 1 \mathrm{H} ; \mathrm{N}=\mathrm{CH}), 7.71(\mathrm{~s}, 1 \mathrm{H}, \mathrm{CH}), 3.61\left(\mathrm{~s}, 3 \mathrm{H}, \mathrm{CH}_{3}\right) ;(\mathrm{m}+1) 251.1\end{array}$ \\
\hline
\end{tabular}


PK5 Yield:68.2\%; $\mathrm{R}_{\mathrm{f}}: 0.62$ \{ethyl acetate: hexane(3:7)\};M.P:140-145 ${ }^{\circ} \mathrm{C} ; \mathrm{IR}\left(\mathrm{KBr} \mathrm{cm}^{-1}\right)$ 1568.59 $(\mathrm{C}=\mathrm{C}), \quad 1656.32(\mathrm{C}=\mathrm{N}), 3112.12(\mathrm{C}-\mathrm{H}), 1242.88(\mathrm{C}-\mathrm{N}), 2857.55(\mathrm{~N}=\mathrm{CH})$, 755.30(C-Cl); NMR: ${ }^{1} \mathrm{H}$ NMR (400MHz, $\left.\mathrm{CDCl}_{3}\right)$ 8:7.19-7.34(m,9H,ArH), 8.52 $(\mathrm{s}, 1 \mathrm{H} ; \mathrm{N}=\mathrm{CH}), 7.60(\mathrm{~s}, 1 \mathrm{H}, \mathrm{CH}) ;(\mathrm{m}+1) 255.1$

PK6 Yield:64.5\%; $\mathrm{R}_{\mathrm{f}}$ : 0.79 ethyl acetate: hexane(3:7)\};M.P:205-210 ${ }^{\circ} \mathrm{C}$; IR $\left(\mathrm{KBr} \mathrm{cm}^{-1}\right)$ 1575.20 $(\mathrm{C}=\mathrm{C}), \quad 1665.67(\mathrm{C}=\mathrm{N}), \quad 3077.79(\mathrm{C}-\mathrm{H}), \quad 1329.45(\mathrm{C}-\mathrm{N}), 2856.61(\mathrm{~N}=\mathrm{CH})$, $3322.97(-\mathrm{OH}) \mathrm{H}$ bonded,1498.71(R-NO $\left.\mathrm{N}_{2}\right)$ NMR: ${ }^{1} \mathrm{H}$ NMR(400MHz, $\left.\mathrm{CDCl}_{3}\right) \delta: 7.71-$ 8.13(m,9H,ArH, 8.49(s,1H;N=CH),7.32(s,1H,CH),6.23(d,1H;ArOH); (m+1) 282.3

PK7 Yield:62.3\%; $\mathrm{R}_{\mathrm{f}}: 0.72\left\{\right.$ ethyl acetate:hexane(3:7)\};M.P:180-185 ${ }^{\circ} \mathrm{C} ; \operatorname{IR}\left(\mathrm{KBr} \mathrm{cm}{ }^{-1}\right)$ 1490.75(C=C), 1675.45(C=N), 3092.12(C-H), 1110.32(C-N), 2846.72(N=CH) 3220.67(-OH) H bonded; NMR: ${ }^{1} \mathrm{H}$ NMR(400MHz, $\left.\mathrm{CDCl}_{3}\right) \delta: 6.75-7.1(\mathrm{~m}, 8 \mathrm{H}, \mathrm{ArH})$, $8.60(\mathrm{~s}, 1 \mathrm{H} ; \mathrm{N}=\mathrm{CH}), 7.4(\mathrm{~s}, 1 \mathrm{H}, \mathrm{CH}), 5.5(\mathrm{t}, 1 \mathrm{H}, \mathrm{ArOH}) ;(\mathrm{m}+1) 237.1$

PK8 Yield:62.7\%; $\mathrm{R}_{\mathrm{f}}: 0.80$ ethyl acetate:hexane (3:7)\};M.P:155-160 ${ }^{\circ} \mathrm{C} ; \mathrm{IR}\left(\mathrm{KBr} \mathrm{cm}^{-1}\right)$ 1530.34(C=C),1647.31(C=N),3087.42(C-H), 1010.11(C-N), $2840.42(\mathrm{~N}=\mathrm{CH})$;

NMR: ${ }^{1} \mathrm{H}$ NMR(400MHz, $\left.\mathrm{CDCl}_{3}\right) \quad \delta: 6.52-7.01(\mathrm{~m}, 9 \mathrm{H}, \mathrm{ArH}), 8.55(\mathrm{~s}, 1 \mathrm{H} ; \mathrm{N}=\mathrm{CH})$, 7.12(s,1H,CH),4.41(s,1H,CH); (m+1) 236.3

PK9 Yield:72.6\%; $\mathrm{R}_{\mathrm{f}}: 0.77\{$ ethyl acetate:hexane $(3: 7)\} ; \mathrm{M} . \mathrm{P}: 145-150{ }^{\circ} \mathrm{C} ; \mathrm{IR}\left(\mathrm{KBr} \mathrm{cm}^{-1}\right)$ 1545.32(C=C), 1652.32(C=N), 3085.51(C-H), 1090.17(C-N), 2842.91 $(\mathrm{N}=\mathrm{CH})$, 1552.31(R-NO $\left.{ }_{2}\right) ; \mathrm{NMR}:{ }^{1} \mathrm{H}$ NMR(400MHz, $\left.\mathrm{CDCl}_{3}\right) \delta: 7.52-7.88(\mathrm{~m}, 9 \mathrm{H}, \mathrm{ArH}), 8.52$ $(\mathrm{s}, 1 \mathrm{H} ; \mathrm{N}=\mathrm{CH}), 7.41(\mathrm{~s}, 1 \mathrm{H}, \mathrm{CH}) ;(\mathrm{m}+1) 266.2$

PK10 Yield:53.3\%; R : 0.65 ethyl acetate: hexane (3:7)\}; M.P:200-205 ${ }^{\circ} \mathrm{C}$; IR $\left(\mathrm{KBr} \mathrm{cm}^{-1}\right)$ 1551.43(C=C), 1672.13(C=N), 3073.62(C-H), 1213.12(C-N), $2850.11(\mathrm{~N}=\mathrm{CH}) 3321$. 51(-OH) H bonded, 1473.91(R-NO $)$; NMR : ${ }^{1} \mathrm{H}$ NMR $\left(400 \mathrm{MHz}, \mathrm{CDCl}_{3}\right) \delta: 7.83-8.13$ $(\mathrm{m}, 8 \mathrm{H}, \mathrm{ArH}), 8.51(\mathrm{~s}, 1 \mathrm{H} ; \mathrm{N}=\mathrm{CH}), 7.32(\mathrm{~s}, 1 \mathrm{H}, \mathrm{CH}), 6.74(\mathrm{~d}, 1 \mathrm{H}, \mathrm{ArOH}) ;(\mathrm{m}+1) 282.1$

PK11 Yield:74.8\%; $\mathrm{R}_{\mathrm{f}}: 0.70$ ethyl acetate: hexane(3:7)\};M.P:130-135 ${ }^{\circ} \mathrm{C} ; \mathrm{IR}\left(\mathrm{KBr} \mathrm{cm}^{-1}\right)$ 1530.42(C=C), 1654.56(C=N), 3092.34(C-H), 1203.56(C-N), 2848.43(N=CH), 1555.10(R-NO $\left.{ }_{2}\right) ; \mathrm{NMR}:{ }^{1} \mathrm{H}$ NMR(400MHz, $\left.\mathrm{CDCl}_{3}\right) \quad \delta: 7.85-8.3(\mathrm{~m}, 9 \mathrm{H}, \mathrm{ArH}), 8.53$ $(\mathrm{s}, 1 \mathrm{H} ; \mathrm{N}=\mathrm{CH}), 7.6(\mathrm{~s}, 1 \mathrm{H}, \mathrm{CH}) ;(\mathrm{m}+1) 266.1$

PK12 Yield:55.5\%; $\mathrm{R}_{\mathrm{f}}: 0.66$ \{ethyl acetate: hexane $\left.(3: 7)\right\} ; \mathrm{M} . \mathrm{P}: 150-155{ }^{\circ} \mathrm{C}$; IR $\left(\mathrm{KBr} \mathrm{cm}^{-1}\right)$ 1562.16(C=C), 1664.31 $(\mathrm{C}=\mathrm{N}), \quad 3175.34(\mathrm{C}-\mathrm{H}), \quad 1092.42(\mathrm{C}-\mathrm{N}), \quad 2845.31(\mathrm{~N}=\mathrm{CH})$ 1642.51(C=O); NMR: ${ }^{1} \mathrm{H} \quad \mathrm{NMR}\left(400 \mathrm{MHz}, \mathrm{CDCl}_{3}\right) \quad \delta: 7.25-7.50(\mathrm{~m}, 5 \mathrm{H}, \mathrm{ArH}), 8.50$ $(\mathrm{s}, 1 \mathrm{H} ; \mathrm{N}=\mathrm{CH}), 7.71(\mathrm{~s}, 1 \mathrm{H}, \mathrm{CH}), 5.71,6.12\left(\mathrm{~s}, 2 \mathrm{H}, \mathrm{NH}_{2}\right) ;(\mathrm{m}+1) 188.0$

PK13 Yield:61.6\%; $\mathrm{R}_{\mathrm{f}}: 0.57$ ethyl acetate:hexane (3:7)\};M.P:140-145 ${ }^{\circ} \mathrm{C} ; \mathrm{IR}\left(\mathrm{KBr} \mathrm{cm}^{-1}\right)$ 1547.23(C=C), 1649.13(C=N), 3082.43(C-H), 1101.38(C-N), 2852.44(N=CH) 3392.12( $\left.\mathrm{R}_{2}-\mathrm{NH}\right) ; \mathrm{NMR}:{ }^{1} \mathrm{H} \mathrm{NMR}\left(400 \mathrm{MHz}, \mathrm{CDCl}_{3}\right)$ 8:6.70-7.17(m,8H,ArH), 8.69 $(\mathrm{s}, 1 \mathrm{H} ; \mathrm{N}=\mathrm{CH}), 7.71(\mathrm{~s}, 1 \mathrm{H}, \mathrm{CH}), 4.65(\mathrm{~s}, 1 \mathrm{H}, \mathrm{ArNH}) ;(\mathrm{m}+1) 236.0$

PK14 Yield:51.7\%; $\mathrm{R}_{\mathrm{f}}: 0.74$ \{ethyl acetate: hexane(3:7)\};M.P:210-215 ${ }^{\circ} \mathrm{C} ; \operatorname{IR}\left(\mathrm{KBr} \mathrm{cm}^{-1}\right)$ 1490.34(C=C), 1674.36(C=N), 3095.65(C-H), 1215.38(C-N), 2857.13(N=CH), 1321.42(C-F); NMR: ${ }^{1} \mathrm{H}$ NMR(400MHz,CDCl3) $\delta: 7.52-7.78(\mathrm{~m}, 9 \mathrm{H}, \mathrm{ArH}), \quad 8.57$ $(\mathrm{s}, 1 \mathrm{H} ; \mathrm{N}=\mathrm{CH}), 7.21(\mathrm{~s}, 1 \mathrm{H}, \mathrm{CH}), 3.54(\mathrm{~s}, 1 \mathrm{H}, \mathrm{ArNH}) ;(\mathrm{m}+1) 254.1$

Antimicrobial activity

The antimicrobial activities of synthesized Schiff bases were evaluated against eleven different strains of microorganism (Five gram positive, Five gram negative bacteria and one fungus) using nutrient agar medium (Hi-Media Laboratories, India) and sabouraud dextrose agar medium (Hi-Media Laboratories, India) respectively. Zone of inhibition of compounds were determined by Cup plate method and minimum inhibitory concentration of the test compounds were determined by two fold serial dilution technique. Dimethyl sulfoxide (DMSO) was used as solvent for both techniques. Paper disc diffusion method for zone of 
inhibition \& minimum inhibitory concentrations of the synthesized compounds were determined by serial dilution method. Ciprofloxacin, ampicilin and fluconazole were used as reference standards for antibacterial and antifungal activity respectively.

The lowest concentration of the test compounds showing no visible microbial growth were considered as minimum inhibitory concentration. The observed zone of inhibition and MIC values for bacterial and fungal strains are given in Table 3 and 4 respectively.

Table 3. Antibacterial activity of the synthesized compounds

\begin{tabular}{|c|c|c|c|c|c|}
\hline \multirow{2}{*}{$\begin{array}{l}\text { Compd. } \\
\text { code }\end{array}$} & \multicolumn{5}{|c|}{ In vitro activity-zone of inhibition in $\mathrm{mm}$, (MIC in $\mu \mathrm{g} / \mathrm{mL}$ ) for gram positive strains } \\
\hline & S. aureus & B. subtilis & B. cereus & P. fluorescence & S. epidermidis \\
\hline PK1 & $14(50)$ & $19(25)$ & $18(50)$ & $16(25)$ & $15(50)$ \\
\hline PK2 & $15(50)$ & $19(25)$ & $14(100)$ & $13(50)$ & $12(100)$ \\
\hline PK3 & $17(25)$ & $14(50)$ & $21(25)$ & $14(50)$ & $13(100)$ \\
\hline PK4 & $18(25)$ & $17(25)$ & $17(50)$ & $13(50)$ & $16(25)$ \\
\hline PK5 & $13(50)$ & $24(6.25)$ & $18(50)$ & $12(50)$ & $18(12.5)$ \\
\hline PK6 & $12(50)$ & $22(12.5)$ & $15(100)$ & $15(25)$ & $16(25)$ \\
\hline PK7 & $22(6.25)$ & $23(6.25)$ & $20(25)$ & $14(50)$ & $14(50)$ \\
\hline PK8 & $12(50)$ & $18(25)$ & $13(100)$ & 11(100) & $14(50)$ \\
\hline PK9 & $15(50)$ & $14(50)$ & $19(50)$ & $14(50)$ & $17(25)$ \\
\hline PK10 & $20(12.5)$ & $20(12.5)$ & 20(25) & $16(25)$ & $10(>100)$ \\
\hline PK11 & $15(25)$ & $16(25)$ & $18(50)$ & $18(12.5)$ & $12(100)$ \\
\hline PK12 & $13(50)$ & 13(50) & $16(100)$ & $12(50)$ & $12(100)$ \\
\hline PK13 & $19(12.5)$ & $22(12.5)$ & $15(100)$ & $14(50)$ & $16(25)$ \\
\hline PK14 & $21(6.25)$ & $18(25)$ & $12(>100)$ & $13(50)$ & $15(50)$ \\
\hline $\begin{array}{l}\text { Ciprofl- } \\
\text { oxacin }\end{array}$ & $25(3.12)$ & $26(3.12)$ & $23(6.25)$ & $21(6.25)$ & $19(12.5)$ \\
\hline \multirow[t]{3}{*}{$\begin{array}{l}\text { Amoxi- } \\
\text { cillin }\end{array}$} & $33(3.12)$ & $34(3.12)$ & $25(6.25)$ & $22(6.25)$ & $20(12.5)$ \\
\hline & \multicolumn{5}{|c|}{ In vitro activity-zone of inhibition in $\mathrm{mm}(\mathrm{MIC}$ in $\mu \mathrm{g} / \mathrm{mL})$ For gram negative strains } \\
\hline & E. coli & P. aeruginosa & K. pneumonae & $P$. vulgaris & S. Typhimurium \\
\hline PK1 & $18(25)$ & $19(25)$ & $13(100)$ & $14(100)$ & $14(100)$ \\
\hline PK2 & $16(50)$ & $16(50)$ & $21(25)$ & $16(50)$ & $18(50)$ \\
\hline PK3 & $15(50)$ & $21(25)$ & $13(100)$ & $11(>100)$ & $10(>100)$ \\
\hline PK4 & $18(25)$ & $20(25)$ & $19(50)$ & $9(>100)$ & $9(>100)$ \\
\hline PK5 & $22(12.5)$ & $17(50)$ & $17(50)$ & $12(100)$ & $17(50)$ \\
\hline PK6 & $19(25)$ & $16(50)$ & $12(100)$ & $13(100)$ & $11(>100)$ \\
\hline PK7 & $21(12.5)$ & $20(25)$ & $17(50)$ & $13(100)$ & $14(100)$ \\
\hline PK8 & $16(50)$ & $13(100)$ & $16(50)$ & $16(50)$ & $15(100)$ \\
\hline PK9 & $15(50)$ & $15(50)$ & $10(>100)$ & $17(50)$ & $17(50)$ \\
\hline PK10 & $16(50)$ & $17(50)$ & $9(>100)$ & $15(50)$ & $13(100)$ \\
\hline PK11 & $12(100)$ & $22(25)$ & $15(50)$ & $10(>100)$ & $16(50)$ \\
\hline PK12 & $14(50)$ & $12(100)$ & $20(25)$ & $15(50)$ & $18(50)$ \\
\hline PK13 & $15(50)$ & $15(50)$ & $18(50)$ & $13(100)$ & $15(100)$ \\
\hline PK14 & $15(100)$ & $14(50)$ & $14(100)$ & $11(>100)$ & $12(>100)$ \\
\hline Ciprofl- & $28(3.12)$ & $32(3.12)$ & $24(6.25)$ & $20(12.5)$ & $21(12.5)$ \\
\hline $\begin{array}{l}\text { Amoxi- } \\
\text { cillin }\end{array}$ & $3(3.12)$ & $24(3.12)$ & $25(6.25)$ & $23(12.5)$ & $20(12.5)$ \\
\hline
\end{tabular}


Table 4. Antifungal activity of the synthesized compounds

\begin{tabular}{cc}
\hline Comp. Code & Zone of inhibition in $\mathrm{mm},(\mathrm{MIC}$ in $\mu \mathrm{g} / \mathrm{mL})$ C. albicans \\
\hline PK1 & $15(50)$ \\
PK2 & $18(25)$ \\
PK3 & $19(25)$ \\
PK4 & $16(50)$ \\
PK5 & $20(12.5)$ \\
PK6 & $13(100)$ \\
PK7 & $18(25)$ \\
PK8 & $17(25)$ \\
PK9 & $17(25)$ \\
PK10 & $15(50)$ \\
PK11 & $12(>100)$ \\
PK12 & $16(50)$ \\
PK13 & $13(100)$ \\
PK14 & $11(>100)$ \\
Fluconazole & $21(12.5)$ \\
\hline
\end{tabular}

\section{Results and Discussion}

We have synthesized a series of Schiff bases of indole-3-aldehyde by microwave assisted synthesis. This protocol presented many advantages, such as good to excellent yields, much shorter reaction time (3-15 $\mathrm{min})$ and simple purification procedure. The bioassay results revealed that most of the synthesized compounds exhibited good antimicrobial activity. Compound (Z)-3-((1H-indol-3-yl) methyleneamino)phenol and $(E)-N 1$ ((1H-indol-3-yl)methylene)-4-fluorobenzene-1,2-diamine showed highest antibacterial activity with MIC of $6.25 \mu \mathrm{g} / \mathrm{mL}$ against $S$. aureus and compound $(E)-N-((1 H$-indol-3yl)methylene)-4-chlorobenzenamine and $(Z)-3-((1 H$-indol-3-yl)methyleneamino) phenol showed highest antibacterial activity with MIC of $6.25 \mu \mathrm{g} / \mathrm{mL}$ against $B$. subtilis. Compound $(E)-N$ - $((1 H$-indol-3-yl)methylene)-4-chlorobenzenamine was found to have highest antifungal activity with MIC of $12.5 \mu \mathrm{g} / \mathrm{mL}$ against $C$. albicans.

\section{Conclusion}

The structures of synthesized compounds were confirmed by IR and NMR spectroscopy. All compounds exhibited significant antibacterial activity but they showed moderate antifungal activity. Further bioassay, optimization and structure-activity relationship of the title compounds are underway.

\section{References}

1. Jerry M, Advanced Organic Chemistry Reactions: Mechanisms and Structure; $4^{\text {th }}$ Ed., John Wiley \& Sons: New York, 1992, 896.

2. Sinha D, Tiwari A K, Singh S, Shukla G, Mishra P, Chandra H and Mishra A K, Eur J Med Chem., 2007, xx, 1-6.

3. Karthikeyan M S, Prasad D J, Poojary B, Bhatt K S, Holla B S and Kumari N S, Bioorg Med Chem., 2006, 14(22), 7482-7489.

4. Patole J, Shingnapurkar D, Padhye S and Ratledge C, Bioorg Med Chem Lett., 2006, 16, 1514-1517. 
5. Vicini P, Geronikaki A, Incerti M, Busonera B, Poni G, Cabras C A and Colla P L, Bioorg Med Chem., 2003, 11, 4785-4789.

6. Sridhar S K, Saravanan M and Ramesh A, Eur J Med Chem., 2001, 36, 615-625.

7. Fioravanti R, Biava M, Porretta G C, Landolfi C, Simonetti N, Villa A, Conte E and Portapuglia A, Eur J Med Chem., 1995, 30, 123-132.

8. Parekh J, Inamdhar P, Nair R, Baluja S and Chanda S J, Serb Chem Soc., 2005, 70 (10), 1155-1161.

9. Pandeya S N, Sriram D, Nath G and DeClercq E, Eur J Pharm Sci., 1999, 9, 25-31.

10. Pandeya S N and Sriram D, Acta Pharmaceutica Turcica, 1998, 40(1), 33-38.

11. Panneerselvam P, Nair R R and Vijayalakshmi G, Eur J Med Chem., 2005, 40(12), 225-229.

12. Pathak P, Jolly V S and Sharma K P, Oriental J Chem., 2000, 16(1), 161-162.

13. Samadhiya S and Halve A, Oriental J Chem., 2001, 17(1), 119-122.

14. Popp F D, J Heterocycl Chem., 1984, 21(2), 617-619. 


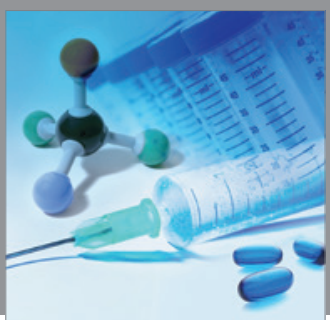

International Journal of

Medicinal Chemistry

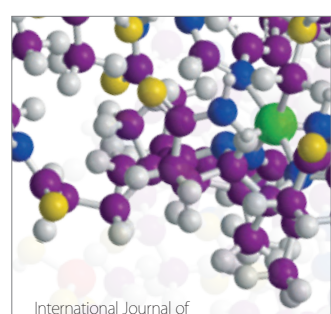

Carbohydrate Chemistry

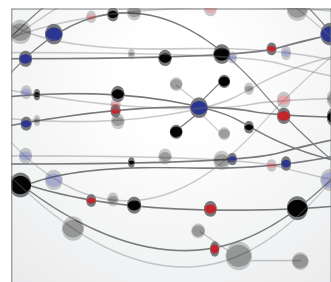

The Scientific World Journal
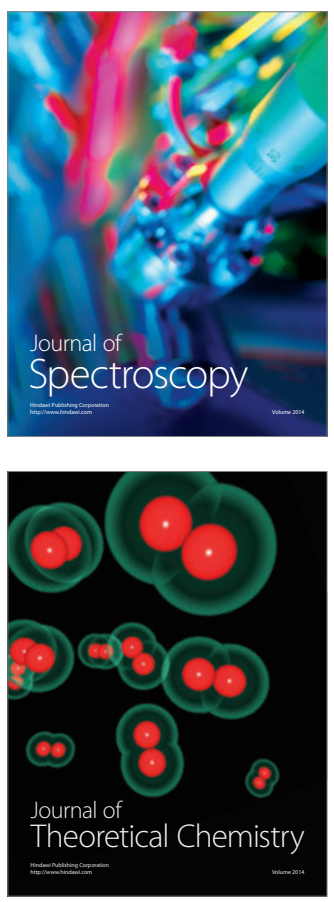
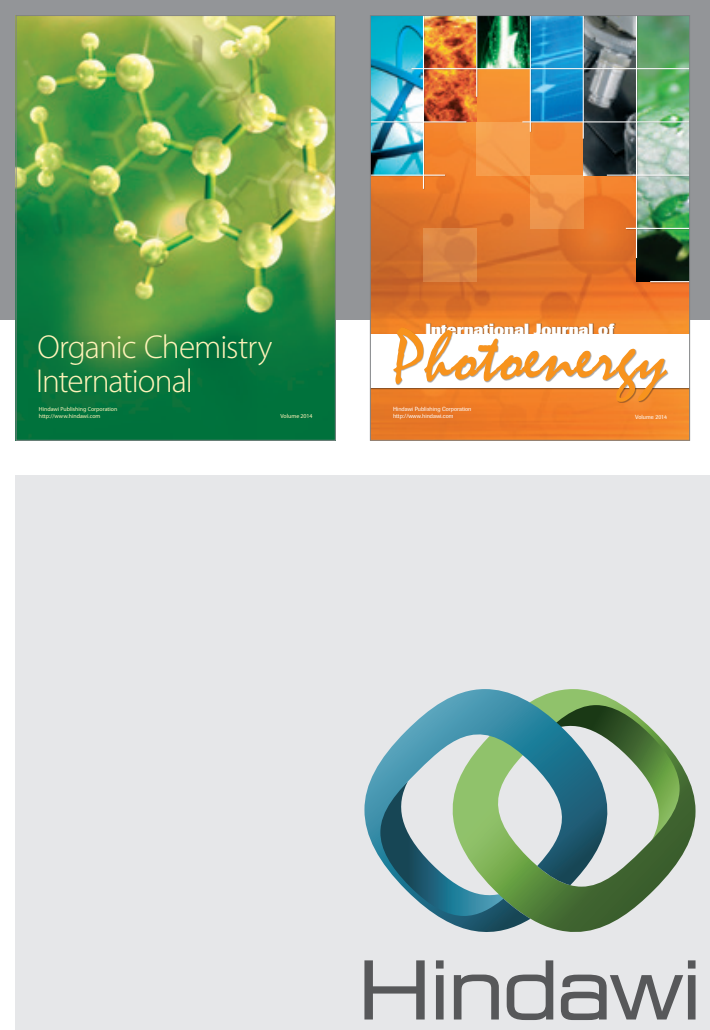

Submit your manuscripts at

http://www.hindawi.com
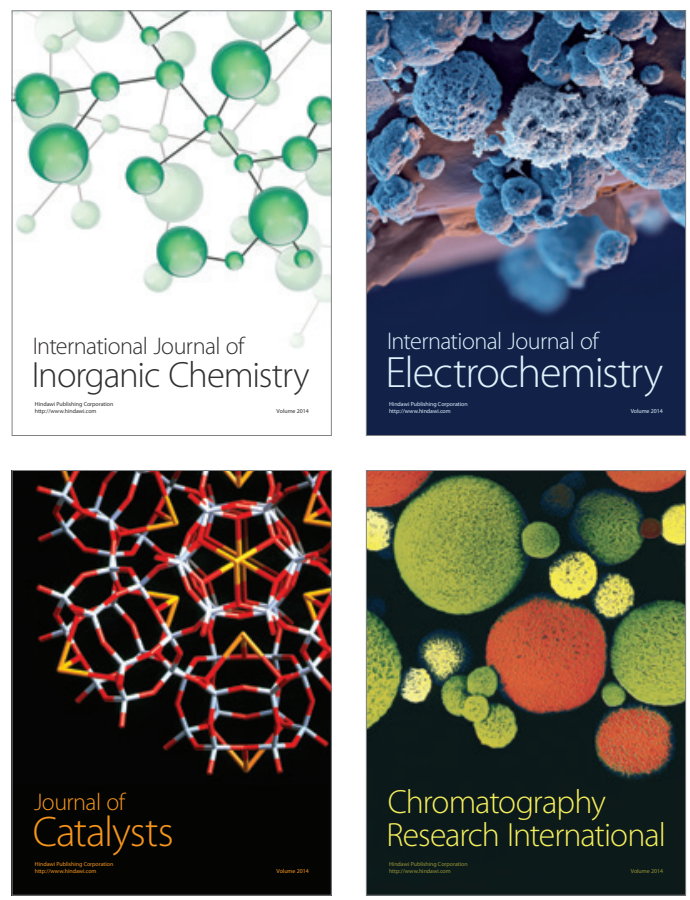
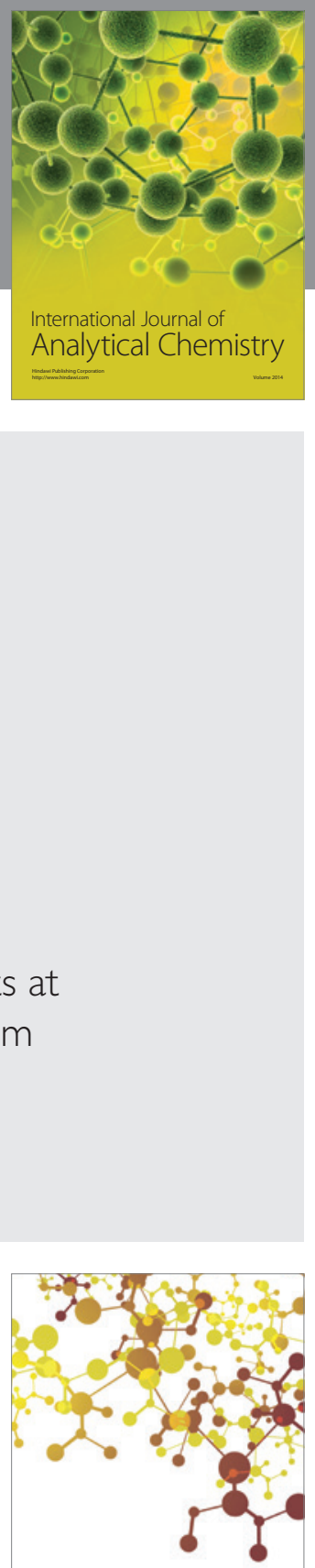

Journal of

Applied Chemistry
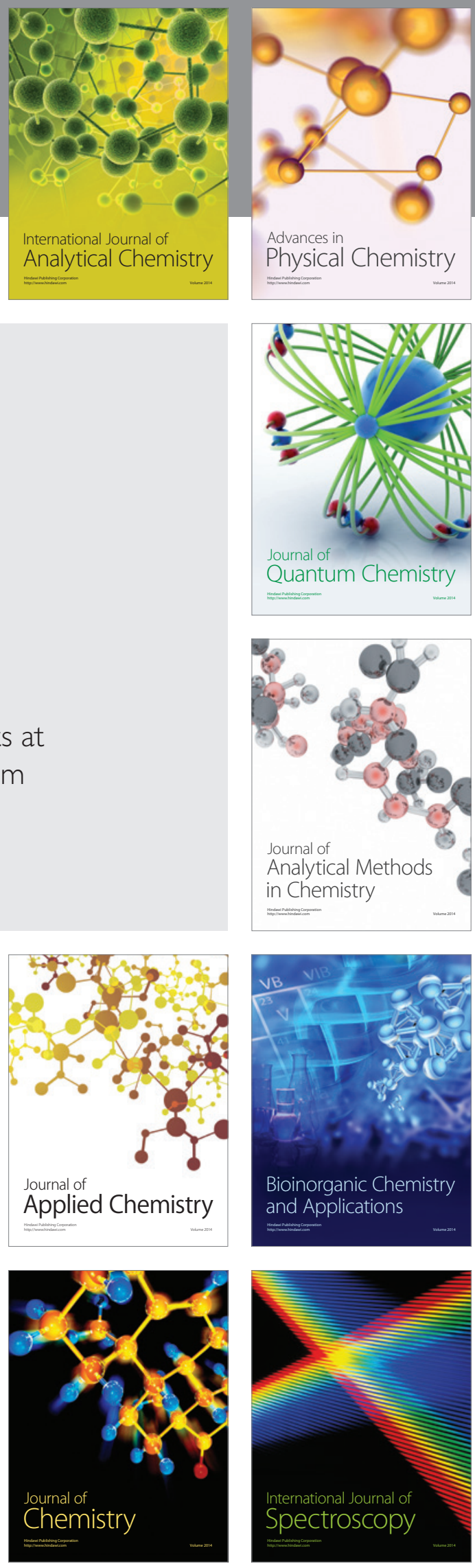